\title{
Modelado de estrategias de labranza agrícola mecanizada para diversos cultivos mediante un algoritmo informático de toma de decisiones
}

\author{
Carlos Roberto Martínez Martínez \\ Ingeniero en Sistemas Informáticos \\ Docente investigador, Facultad de Ingeniería y Arquitectura \\ Universidad Católica de El Salvador, El Salvador \\ carlosm2@gmail.com
}

Recepción: 27/06/2016

Aceptación: 08/07/2016

\section{Resumen}

Considerando que la documentación aplicada a técnicas de labranza agrícola en El Salvador es limitada, pese al hecho que éstas son fundamentales para el establecimiento y éxito de cultivos que tienen fines de explotación económica, se desarrolló un algoritmo que fue capaz de elaborar planes de labranza con el fin de apoyar la toma de decisiones en dicho campo. En la preparación de suelos influyen muchas variables que interaccionan entre sí, tales como los distintos niveles de humedad, presencia de capas rocosas a diferentes profundidades, la textura del suelo y la profundidad de su capa fértil, los tipos de maquinaria, la extensión del área a cultivar, entre otros. La combinación matemática de todas estas variables dio como resultado una cantidad muy alta de opciones, por lo cual se estimó que es alta la posibilidad de que los cultivos tengan problemas dados el desconocimiento sobre el uso de maquinaria agrícola. El algoritmo llamado SIMA, por sus siglas de Simulador de Mecanización Agrícola fue dotado de una interfaz web desde la cual el usuario puede introducir las condiciones del campo; y el software, por su parte, fue capaz de realizar las validaciones y cálculos necesarios para proveer de un plan de labranza eficiente y confiable. Los servicios de dicho algoritmo están disponibles para los usuarios de internet a través del sitio web http://www.catolica.edu.sv/decanatos/iya/sima/.

Palabras clave: software educativo, labranza, agronomía, cultivos, aprendizaje, toma de decisiones.

\begin{abstract}
Considering that the documentation applied to agricultural tillage techniques in El Salvador is limited, despite the fact that these are fundamental for the establishment and success of crops that have economic exploitation purposes, an algorithm was developed that was able to elaborate tillage plans with In order to support decision-making in the field. In soil preparation, many variables interact, such as different levels of humidity, presence of rock layers at different depths, soil texture and depth of fertile layer, types of machinery, extension of the area To cultivate, among others. The mathematical combination of all these variables resulted in a very high number of options, so it was estimated that the possibility that crops have problems due to lack of knowledge about the use of agricultural machinery is high. The algorithm called SIMA, by its initials of Simulator of Agricultural Mechanization was endowed with a web interface from which the user can introduce the conditions of the field; And the software, for its part, was able to perform the validations and calculations necessary to provide an efficient and reliable tillage plan. The services of this algorithm are available to Internet users through the website http://www.catolica.edu.sv/decanatos/iya/sima/.
\end{abstract}

Key words: educational software, farming, agronomy, crops, learning, decision taking. 


\section{Introducción}

El propósito de la labranza es preparar el suelo para el cultivo, volviéndolo más suave, aireándolo e incorporando materia orgánica. Tradicionalmente esta preparación se realiza empleando un arado, que penetra en el suelo y voltea la tierra, arrancando o eliminando las malas hierbas que crecen en el terreno; removiendo y aflojando las capas superficiales del suelo y dejando un lecho con la humedad suficiente para que germinen las semillas sembradas. Sin embargo, antes de labrar un suelo, hay que definir una estrategia apropiada, ya que se puede perjudicar al suelo si se practica con profundidad y frecuencia inapropiadas, sobre todo si la capa fértil de la superficie es delgada (Chaves, 2006 y Rivera, 2015). Lo ideal sería seguir un programa de labranza mínima o reducida para conservar el suelo, para que la materia vegetal muerta que queda tras la cosecha quede a nivel superficial o bien bajo tierra, a poca profundidad, en vez de ser introducida profundamente con el arado, como ocurre en la labranza tradicional; ello contribuye a mantener la humedad en el interior y a proteger el suelo de la erosión.

Para satisfacer las necesidades actuales de la agricultura, la labranza hoy en día debe ser mecanizada, por lo cual hay que tener conocimiento sobre los distintos implementos que se deben utilizar y sus funciones, así como el tipo de máquina que debe ser acoplada para efectuar la tracción. Una estrategia eficiente de labranza, preservaría el suelo, ahorraría desembolsos económicos y contribuiría al uso mínimo-necesario de combustible diesel para la operación general.

El desconocimiento de cómo labrar eficazmente previo la siembra, puede contribuir a su deterioro. Una mala labranza puede echar a perder la capa fértil del suelo al revolverla con horizontes más profundos, o bien puede ser erosionada por el viento o la lluvia. Las buenas estrategias deben considerar diversidad de factores como el tipo de suelo, época del año, implementos disponibles, el cultivo a establecer, la potencia de tracción requerida, la humedad del suelo, la cantidad de materia orgánica presente, la inclinación y rocosidad del suelo, la cantidad de pasos a efectuar con el fin de ahorrar costos y disminuir el consumo de combustible, entre otros factores.

En el campo salvadoreño, este conocimiento se ha adquirido de forma empírica, de manera que no se ha documentado ampliamente la temática de acuerdo a las condiciones nacionales. En ocasiones, ni siquiera es debidamente trasmitido entre las personas, razón por la cual muchos agricultores han cometido errores que por antonomasia son muy difíciles de corregir. Por esta razón es prioritario saber trabajar bien la tierra mediante el uso apropiado de maquinaria (Boroto, 2005; Alvarado, 2011 y Mendoza 2015). De ahí la 
importancia de crear una herramienta informática que oriente a los nuevos profesionales de la agronomía y a agricultores, sobre esta temática. Al crear un banco de datos con todas las variables que se interrelacionan, es posible desarrollar un algoritmo informático que efectúe un proceso de toma de decisiones y ofrezca como resultado el conjunto de pasos a seguir para preparar adecuadamente la tierra previo al establecimiento de un cultivo, de acuerdo a las condiciones que se tienen en El Salvador.

Por tal motivo, dentro de la investigación se desarrolló un algoritmo informático, bajo el nombre Simulador de Mecanización Agrícola $(\text { SIMA })^{1}$, implementado a través de una interfaz web, que procesa todas esas variables y ofrece planes eficientes de labranza a usuarios de Internet. Tal herramienta servirá no sólo de orientación para personas que aún no poseen pericia sobre el tema, sino que constituirá una herramienta importante que podría catalogarse, además, bajo la rama de Software Educativo, el cual tiene un claro impacto positivo en el proceso de formación académica $\mathrm{y}$ de transferencia de conocimiento (Fuentes, 2006; Reis, 2007; Glusac, 2008; Schez, 2012 y Martínez, 2015).

\section{Materiales y métodos}

Esta investigación fue experimental, con variables cuantitativas, y se desarrolló en las siguientes etapas:

\section{Diagnóstico y recopilación de técnicas de} labranza: Mediante guías técnicas y entrevistas a expertos, se documentaron las técnicas más apropiadas de labranza agrícola para cada tipo de cultivo.

Creación de banco de datos: Se desarrolló un modelo virtual con toda la información recolectada y las reglas de interrelación entre las variables.

Programación del algoritmo: Se desarrolló el software que tomará decisiones y elaborará una estrategia de labranza agrícola según los reconocidos en el diagnóstico.

Elaboración de interfaz visual: Se creó una interfaz web para consumir los servicios del algoritmo y ponerlos a disposición de usuarios de Internet, en forma sencilla.

Validación del modelo: El modelo fue puesto a juicio de conocedores del tema, para comprobar su precisión y exactitud.

\section{Resultados y discusión}

A continuación, se presentan los resultados obtenidos de las fases de diagnóstico, diseño, desarrollo y prueba del algoritmo, así como también de la interfaz web que se creó para que sus servicios pudieran ser consumidos por los usuarios de Internet.

1. El autor también se referirá a este software por medio de su sigla. 


\section{a. Variables de labranza reconocidas}

Sistematizar las técnicas de labranza utilizadas en El Salvador implica tener en consideración gran cantidad de variables, entre las cuales podrían mencionarse:

- Doce tipos de suelo, según la Universidad de Wisconsin-Madison (2010). (Arcilloso, arcillo arenoso, franco arcilloso, arcillo limoso, franco arcillo arenoso, franco arcillo limoso, arena, arena poco franca, franco arenoso, franco, franco limoso, limoso).

- Cinco niveles de humedad del suelo (sobre saturación, saturación, capacidad de campo, punto de marchitez y punto de marchitez permanente). (Instituto Canadiense de Fosfato y Potasa, 2006).

- Tres niveles de profundidad del suelo agrícola (superficial, medio y profundo).

- Tres niveles de cantidad de materia orgánica (Abundante, media y escasa).

- Tres niveles de rocosidad (profunda, media y superficial).

- Tres labranzas por cultivo, como mínimo (roturación, pulimento y siembra).
- Veintidós tipos diferentes de implementos con los que se dispone en El Salvador (Arado profundo, medio y liviano. Rastra pesada, media y liviana. Subsolador de uno, dos y tres cinceles. Fertilizadora media y grande. Rastrillo. Ampliador. Rotovator. Sembradora de grano. Compactador. Surqueador. Nivelador de suelo. Encamadora. Sprayer. Chapodadora. Vertedera mediana y profunda).

- Tamaño del terreno a asistir.

- Tres dimensiones de tractor de acuerdo a su potencia (Pequeño: hasta $75 \mathrm{HP}$. Mediano: entre 80 a 110 HP. Grande: de 120 HP en adelante).

- Cuatro tipos de tracción: rodaje sencillo $2 \mathrm{WD}$, rodaje sencillo $4 \mathrm{WD}$, doble rodaje $2 \mathrm{WD}$, doble rodaje $4 \mathrm{WD}$.

- Al menos nueve tipos de cultivo (Caña de azúcar. Arroz. Maicillo. Maíz y frijol de secano, de regadío y de humedad. Hortalizas.)

Desde una perspectiva de conjunto, las posibilidades de interrelación de todas esas variables, arrojan 13 millones 122 mil combinaciones posibles.

(12 tipos de suelo) $\mathrm{x}(5$ niveles de humedad $) \mathrm{x}(3$ profundidades $) \mathrm{x}$

( 3 cantidades de materia orgánica) $\mathrm{x}$

(3 tipos de rocosidad) $\mathrm{x}$

(3 labranzas) $\mathrm{x}$ (25 implementos) $\mathrm{x}$

(3 potencias de tractor) $\mathrm{x}$

(9 tipos de cultivo) $\mathrm{x}$ (4 tipos de tracción)

Figura 1. Posibilidades de interacción entre variables que intervienen en la labranza agrícola mecanizada. 
La clave a esta astronómica cifra se encuentra en conocer las reglas que determinan cuáles combinaciones no son posibles y cuáles son óptimas. Para sortear estas complejas relaciones, los agricultores se guían por la tradición para conocer cómo realizar sus trabajos, aunque no libres de errores por desconocimiento técnico. En suma, puede decirse que el algoritmo propuesto constituye un modelo de toma de decisiones para la definición de estrategias de labranza.

Para ilustrar la importancia de un conocimiento estratégico de labranzas, se ofrece el siguiente ejemplo: A finales del mes de noviembre del 2015, un grupo de agricultores de la zona de Zapotitán, deseaban establecer un cultivo de frijol por humedad residual. Sin embargo, las lluvias tardías habían provocado la saturación de humedad en el suelo, lo cual no es conveniente para dicho cultivo. No era posible esperar que el suelo secara natural- mente, ya que el momento oportuno de siembra se hubiese perdido, por lo cual se recurrió a efectuar una estrategia de cuatro labranzas para la preparación del suelo. Primero se utilizó un tractor de 110HP, de doble transmisión, con una rastra arado mediana, para romper el suelo mojado y producir escombros de arcilla que estarían expuestos al sol y al viento con el fin de que se secaran (Figura 2-A). Luego de esperar tres días, usando un tractor de $90 \mathrm{HP}$, se hizo una nueva labranza con la misma rastra, con el fin de reducir los escombros a otros más pequeños, los cuales tuvieron otros dos días de secado (Figura 2-B). Luego de ello, con el mismo tractor se hizo un paso de rastra pulidora con el fin de pulverizar el suelo (Figura 2-C), reteniendo así la humedad del subsuelo por capilaridad, siendo esta la condición ideal para sembrar frijol para producción de semilla.
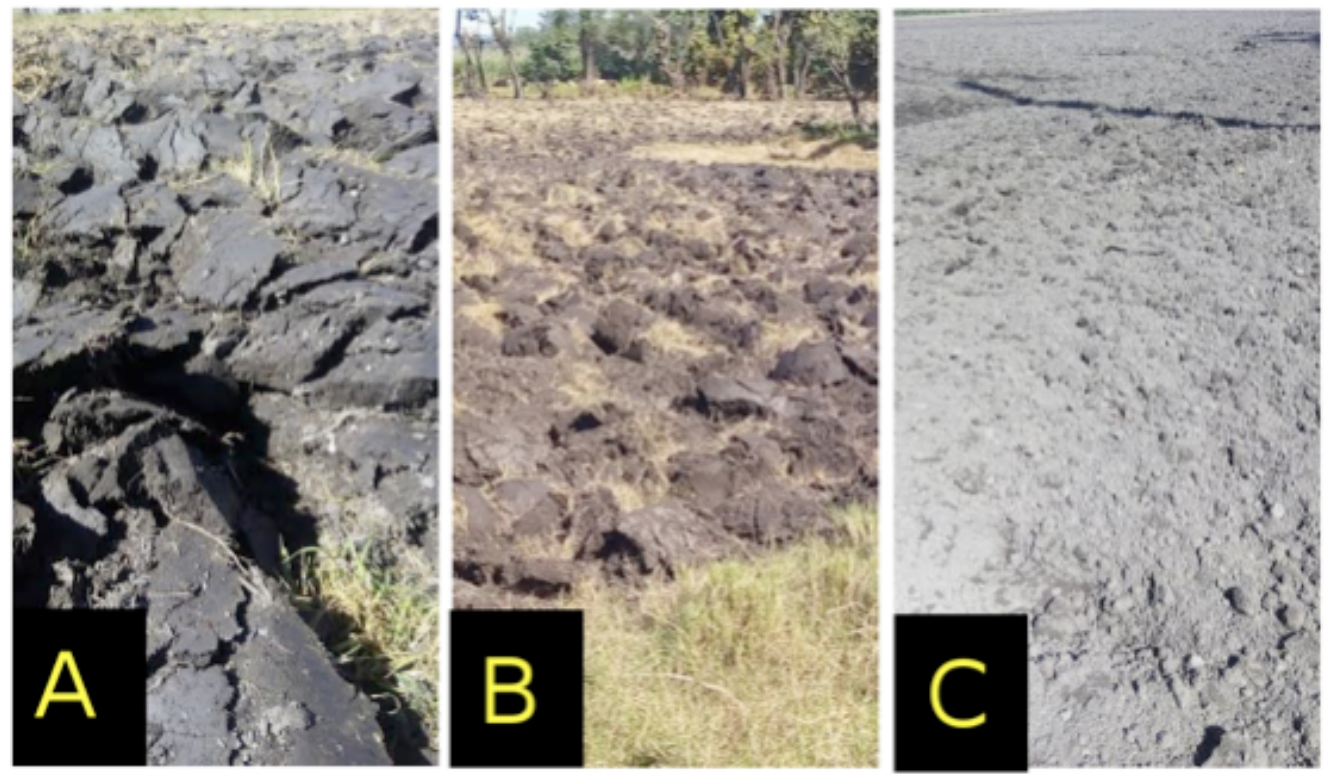

Figura 2. Diferentes resultados de labranza agrícola dependiendo de las condiciones del suelo y de la maquinaria utilizada. 


\section{b. Desarrollo del software}

Dadas las doce siguientes entidades:

- $\mathrm{C}$ = tipo de cultivo que se realiza en El Salvador.

- $\mathrm{L}=$ tipo de labranza efectuada en El Salvador.

- I = tipo de implemento agrícola disponible en El Salvador.

- $\mathrm{T}=$ tipo de tractor disponible en El Salvador.

- $\mathrm{S}=$ tipo de suelo.

- $\mathrm{M}=$ cantidad de materia orgánica presente en el suelo al momento de la labranza.

- $\mathrm{P}$ = profundidad del suelo agrícola (capa cultivable).
- A = categorización de la compactación del suelo.

- $\mathrm{R}=$ profundidad a la cual se encuentra la capa rocosa del suelo.

- $\mathrm{H}=$ humedad del suelo al momento de efectuar la labranza.

- $\mathrm{E}=$ extensión del terreno a cultivar.

- $\mathrm{D}=$ dureza del suelo.

Se creó una base de datos que contiene todas las relaciones viables entre las variables del modelo:

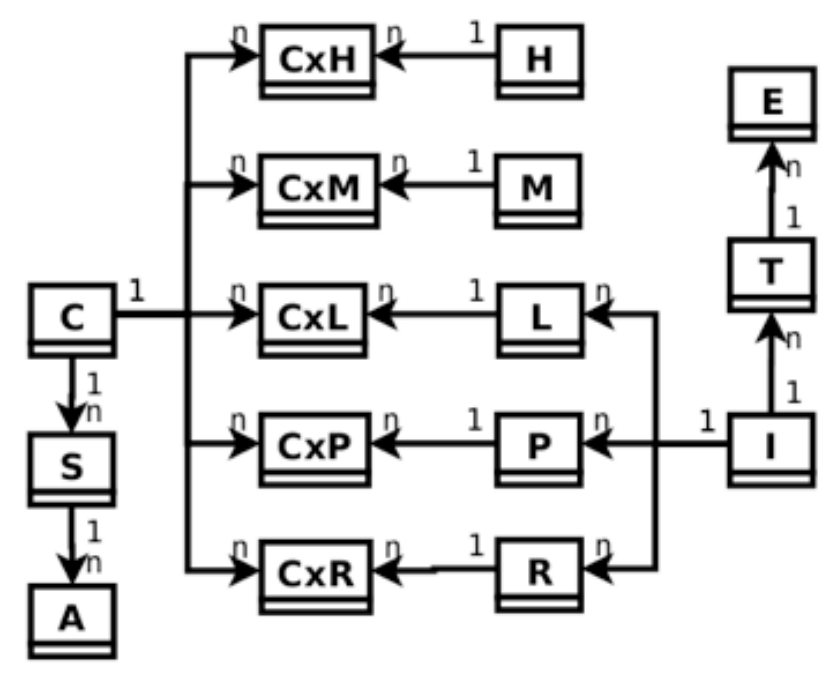

Figura 3. Diagrama Entidad-Relación en el que se modela la base de datos desarrollada.

$\mathrm{Al}$ ejecutarse el algoritmo, en primer lugar, se validó el modelo ingresado por el usuario en la interfaz web, tal como se muestra a continuación, usado la notación de álgebra relacional. El sub índice "v" significa que el conjunto de datos ha sido validado.

$$
\begin{gathered}
\mathrm{CV}_{V}=(\mathrm{C}|\mathrm{x}| \mathrm{S}) \cap(\mathrm{CxH} \cap \mathrm{CxL} \cap \mathrm{CxM} \cap \mathrm{CxP} \cap \mathrm{CxR}) \\
\mathrm{A}_{\mathrm{V}}=\mathrm{S}|\mathrm{x}| \mathrm{A} \\
\mathrm{LV}_{\mathrm{V}}=\mathrm{A} \mathrm{V}|\mathrm{x}| \mathrm{L}|\mathrm{x}| \mathrm{CV}_{\mathrm{V}} \\
\mathrm{I}_{\mathrm{V}}=\mathrm{P}|\mathrm{x}| \mathrm{I}|\mathrm{x}| \mathrm{R}|\mathrm{x}| \mathrm{H}|\mathrm{x}| \mathrm{L}_{\mathrm{V}} \\
\mathrm{T}_{\mathrm{V}}=\mathrm{T}|\mathrm{x}| \mathrm{E}|\mathrm{x}| \mathrm{IV}_{\mathrm{V}}
\end{gathered}
$$


Se determinó el tipo de implemento usable para el trabajo:

$$
\begin{gathered}
\mathrm{I}_{\text {posible }}=(\mathrm{IV}-(\mathrm{CV}|\mathrm{x}| \mathrm{LV})) \\
\text { Iusable }=\mathrm{I}_{\text {posible }}-\mathrm{T}_{\mathrm{V}}
\end{gathered}
$$

Luego, se calculó la dureza del suelo:

$$
\mathrm{D}=\mathrm{S}_{\text {dureza_nominal }}-\mathrm{H}+\mathrm{Av}
$$

Conocidos tales valores, es posible crear una ecuación que simule la creación de un algoritmo predictivo de labranza para la roturación y pulido del suelo:

$$
\begin{aligned}
& \text { Ilabranza }=\mathrm{I}_{\text {usable }}=(\text { arados }+ \text { subsoladores }+ \text { rastras }) \\
& \operatorname{Plan}_{\text {inicial }}={ }_{\mathrm{D}} \sum^{\mathrm{i}=\text { Sdureza } \_ \text {nominal }}\left(\mathrm{D}-\left(\mathrm { CV } _ { | \mathrm { x } | } \left(\mathrm{S}_{|\mathrm{x}|}\right.\right.\right. \\
& \text { (labranza) })+\mathrm{M} \text { ) }
\end{aligned}
$$

Finalmente se agregaron los implentos que complementan la labranza:

Planfinal $=$ Planinicial $+(\mathrm{LV}|\mathrm{x}|$ Iusable $<>($ ar ados+subsoladores+rastras))

El procesamiento de información del modelo fue de carácter secuencial debido a que el que el filtrado y cálculo de datos en cada paso, era insumo directo para las rutinas que le eran subsecuentes. Una representación simplificada del flujo de la información se presenta en la figura 4.

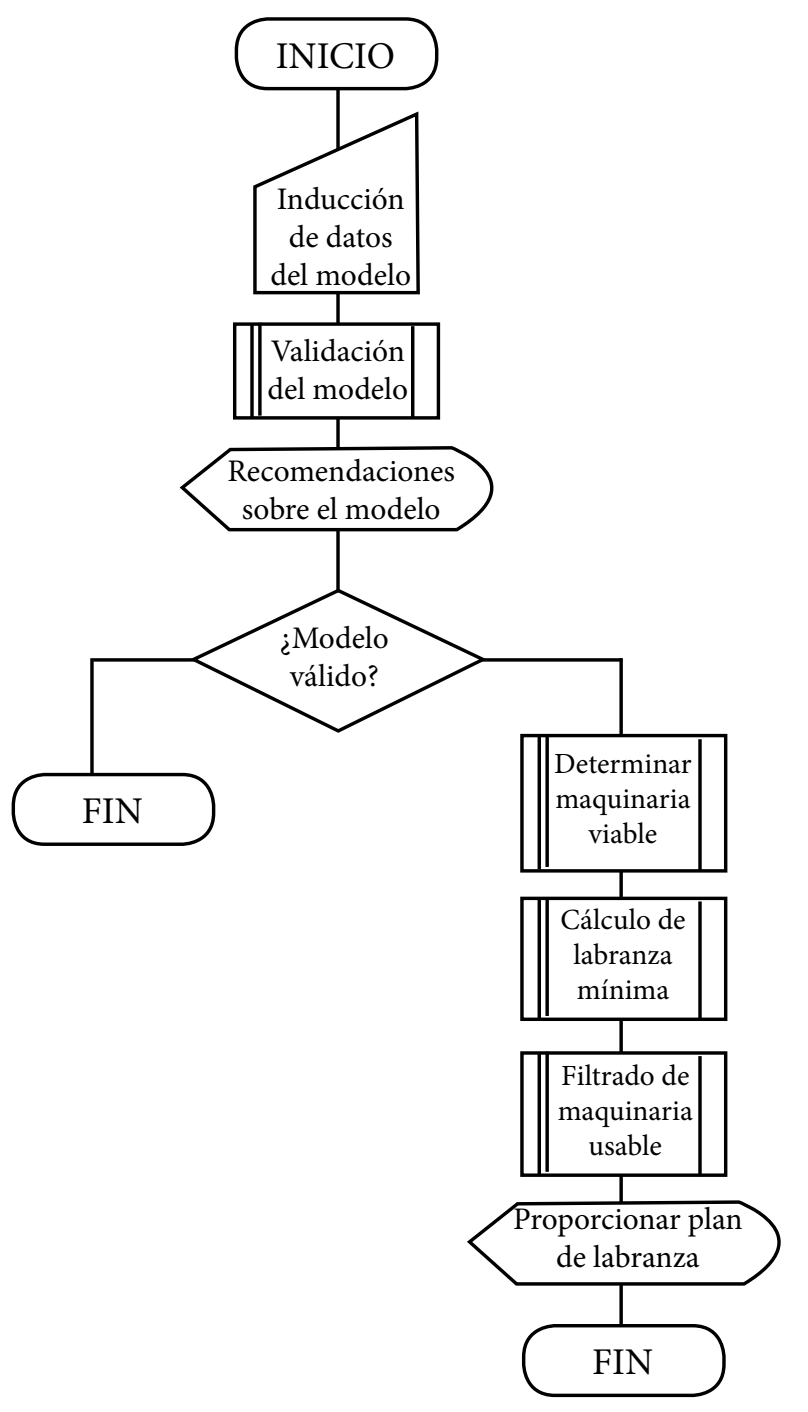

Figura 4. Diagrama de flujo (resumido) de información en el algoritmo.

El algoritmo funciona validando y calculando un plan de labranza de acuerdo con las variables que el usuario haya ingresado, para ello fue necesario proveer de una interfaz visual de carácter adaptativo que fuera fácil de comprender para cualquier usuario de Internet y de teléfonos móviles. La figura 5 muestra la primera parte del formulario de captura de datos. 


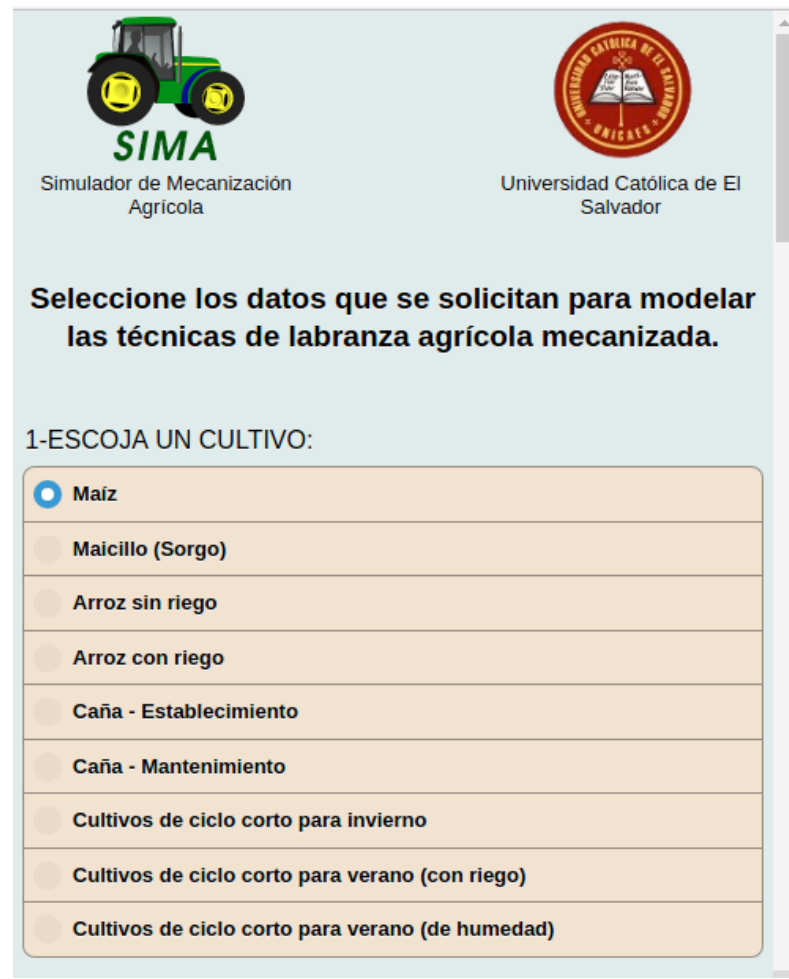

Figura 5. Interfaz visual para ingresar variables al algoritmo.

El usuario debía seleccionar una opción en cada una de las categorías: Tipo de cultivo, tipo de suelo, humedad del suelo, nivel al que se encuentra la capa rocosa del suelo, profundidad de la capa fértil, cantidad de materia orgánica, compactación del suelo y extensión del terreno a labrar. Luego de validar si las relaciones entre las opciones seleccionadas son compatibles, se realizan los cálculos necesarios para ofrecer un plan de labranza explicado en lenguaje común. La figura 6 es un ejemplo de un plan de labranza de maíz, donde las condiciones de compactación ingresada son máximas, la extensión de terreno considerable, y la cantidad de biomasa (ya sea por residuos de cosecha, por malezas acumuladas o ambos) son muy abundantes. Eso da como resultado, uno de los escenarios más

\section{PLAN RECOMENDADO DE LABRANZA:}

1. Debe hacerse labranza profunda con: Subsolador de 3 cinceles

2. Cantidad de labranzas para roturar: $\mathbf{2}$, usando Rastra pesada (de tiro).

3. Espere la aparición de las primeras lluvias para que se ablande la tierra y emerjan las malezas. Con ello, la labranza tendrá doble beneficio.Se recomienda pulir el suelo con el siguiente número de labranzas: $\mathbf{2}$, usando Rastra liviana (de tiro).

4. Efectúe surqueo justo después de pulir, si la siembra será a mano. Si la siembra será con máquina, opcionalmente podría aporcar con el surqueador, cuando la milpa tenga al menos $20 \mathrm{~cm}$ de altura; en este caso, la habilidad del operario del tractor es crucial para no dañar el cultivo.

5. Si el suelo no está muy blando, puede usarse un tractor de Ilanta angosta con boom, para fumigar herbicidas post/pre emergentes en el surqueado, o fertilizantes foliares + insecticida cuando la milpa esté aún pequeña y el tractor no la dañe al pasar.

6. La cosecha se obtiene mediante máquina desgranadora conectada al toma de fuerza de un tractor pequeño, entre 60 a $75 \mathrm{hp}$, nunca mayor a $95 \mathrm{hp}$. Tractores más grandes poseen una potencia sobredimensionada para dicha labor $y$ consumen mucho combustible, lo cual no los hace rentables para tales usos. Otra alternativa es un motor diesel tipo estacionario, que se acopla a una desgranadora y se transporta mediante pick-up y trailer.

7. Para aumentar el valor de mercado de la cosecha, se recomienda pasar el grano por una máquina Sopladora/clasificadora

Figura 6. Presentación de un plan de labranza. 


\section{c. Análisis de eficacia del software}

Una estrategia práctica para validar si los resultados del algoritmo se apegan a la forma óptima en que debe labrarse los cultivos en los suelos salvadoreños, fue presentar el software ante un grupo de gente experta en dicha área. Mediante instrumentos de cotejo, se recopiló y promedió el criterio de los evaluadores, quienes consideraron la adecuación de los resultados a las condiciones configuradas según todas las variables de los modelos ingresados. La tabla 1 muestra los promedios de puntuaciones del algoritmo según sus resultados para cada tipo de suelo, y los tipos de cultivo según se listan a continuación:
A. Maíz (cultivado en forma tradicional, al establecimiento de la temporada lluviosa)

B. Maicillo (sorgo)

C. Arroz con riego.

D. Arroz sin riego.

E. Caña de azúcar (establecimiento)

F. Caña de azúcar (mantenimiento)

G. Cultivos de ciclo corto para invierno (como pepino, tomate, ejote).

H. Cultivos de ciclo corto para verano, mediante riego.

I. Cultivos de ciclo corto para verano, mediante humedad residual del suelo.

Tabla 1. Resumen estadístico de la efectividad del algoritmo y sus variables en términos de cultivo versus tipo de suelo

Efectividad ponderada de planes de labranza para cultivos

\begin{tabular}{|l|c|c|c|c|c|c|c|c|c|c|}
\hline \multicolumn{1}{|c|}{ Suelo } & A & B & C & D & E & F & G & H & I & Promedio \\
\hline Arcilloso & $99 \%$ & $96 \%$ & $100 \%$ & $99 \%$ & $96 \%$ & $96 \%$ & $98 \%$ & $95 \%$ & $98 \%$ & $97 \%$ \\
\hline Arcillo arenoso & $98 \%$ & $96 \%$ & $95 \%$ & $98 \%$ & $98 \%$ & $98 \%$ & $95 \%$ & $97 \%$ & $97 \%$ & $97 \%$ \\
\hline Franco arcilloso & $99 \%$ & $100 \%$ & $100 \%$ & $100 \%$ & $95 \%$ & $97 \%$ & $99 \%$ & $100 \%$ & $98 \%$ & $99 \%$ \\
\hline Arcillo limoso & $100 \%$ & $97 \%$ & $100 \%$ & $96 \%$ & $99 \%$ & $98 \%$ & $98 \%$ & $98 \%$ & $97 \%$ & $98 \%$ \\
\hline $\begin{array}{l}\text { Franco arcillo } \\
\text { arenoso }\end{array}$ & $95 \%$ & $96 \%$ & $95 \%$ & $99 \%$ & $98 \%$ & $100 \%$ & $98 \%$ & $95 \%$ & $100 \%$ & $97 \%$ \\
\hline $\begin{array}{l}\text { Franco arcillo } \\
\text { limoso }\end{array}$ & $99 \%$ & $99 \%$ & $99 \%$ & $96 \%$ & $99 \%$ & $98 \%$ & $97 \%$ & $100 \%$ & $96 \%$ & $98 \%$ \\
\hline Arena & $100 \%$ & $98 \%$ & $100 \%$ & $98 \%$ & $97 \%$ & $98 \%$ & $98 \%$ & $99 \%$ & $95 \%$ & $98 \%$ \\
\hline $\begin{array}{l}\text { Arena poco } \\
\text { franca }\end{array}$ & $98 \%$ & $100 \%$ & $99 \%$ & $97 \%$ & $99 \%$ & $98 \%$ & $99 \%$ & $99 \%$ & $98 \%$ & $99 \%$ \\
\hline \begin{tabular}{l} 
Franco arenoso \\
\hline Franco
\end{tabular} & $99 \%$ & $98 \%$ & $98 \%$ & $99 \%$ & $95 \%$ & $99 \%$ & $99 \%$ & $97 \%$ & $95 \%$ & $98 \%$ \\
\hline Franco limoso & $96 \%$ & $99 \%$ & $97 \%$ & $95 \%$ & $98 \%$ & $97 \%$ & $96 \%$ & $96 \%$ & $95 \%$ & $97 \%$ \\
\hline Limoso & $100 \%$ & $97 \%$ & $98 \%$ & $99 \%$ & $95 \%$ & $97 \%$ & $96 \%$ & $100 \%$ & $95 \%$ & $97 \%$ \\
\hline Promedio global & $99 \%$ & $98 \%$ & $98 \%$ & $98 \%$ & $97 \%$ & $98 \%$ & $97 \%$ & $98 \%$ & $97 \%$ & $98 \%$ \\
\hline
\end{tabular}


El promedio global en cuanto a la precisión de los resultados, según los expertos, fue de $98 \%$. En la figura 7 se grafican los resultados por tipo de suelo y en la figura 8 por cultivo. Los resultados se acercan bastante al 100\% de satisfacción.

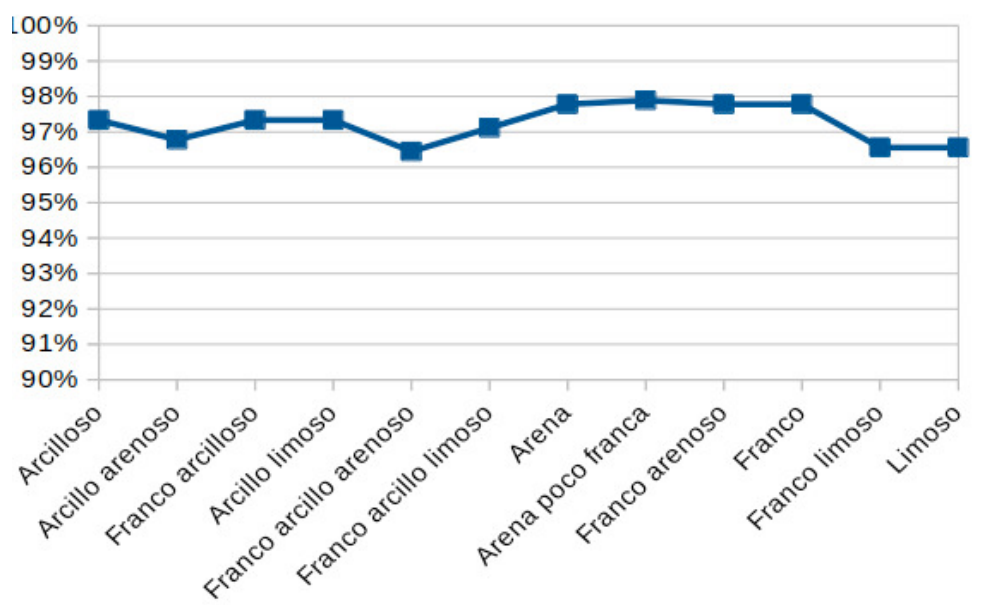

Figura 7. Gráfica de precisión de resultados del algoritmo, según tipos de suelo.

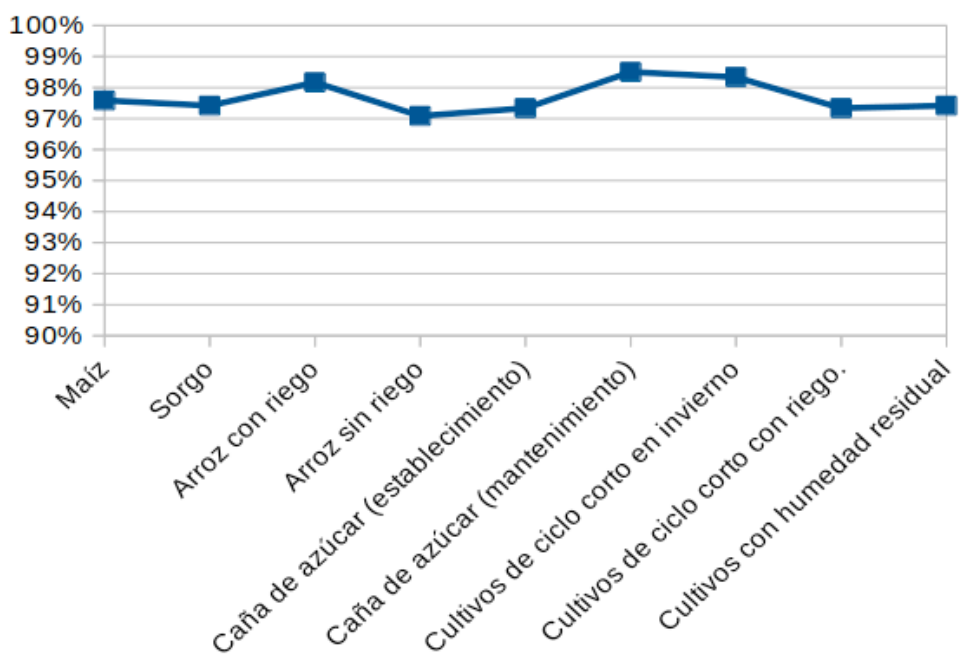

Figura 8. Gráfica de precisión de resultados del algoritmo, según tipos de cultivo.

Los datos del mismo instrumento se usaron como insumo para comparar y establecer una relación cruzada entre variables (Tabla 2).
Como es de esperarse, al promediar los mismos datos, se obtiene una valoración global del $98 \%$. 

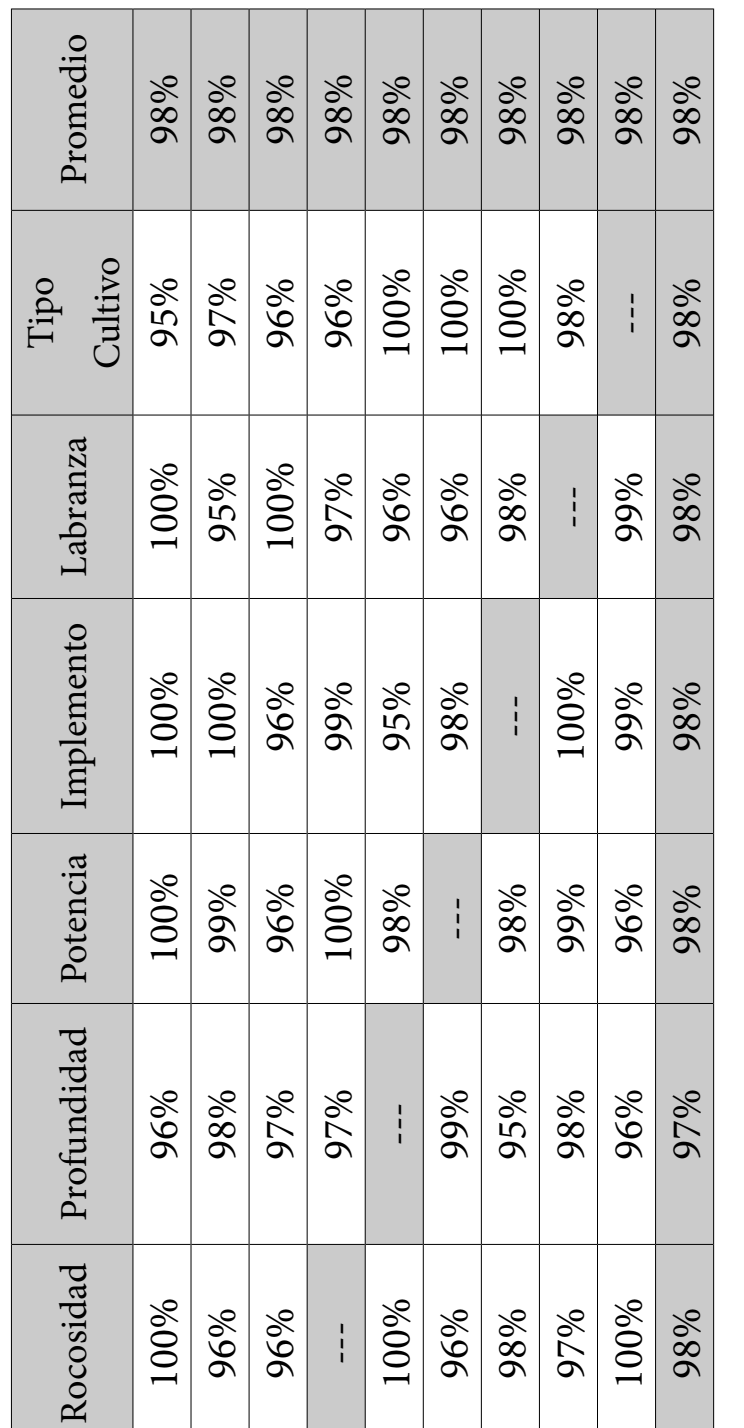

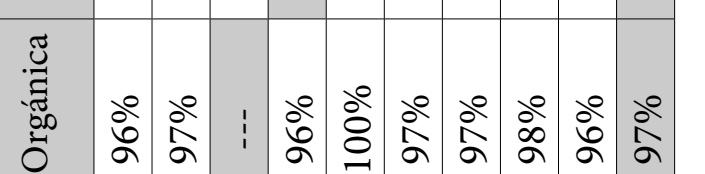

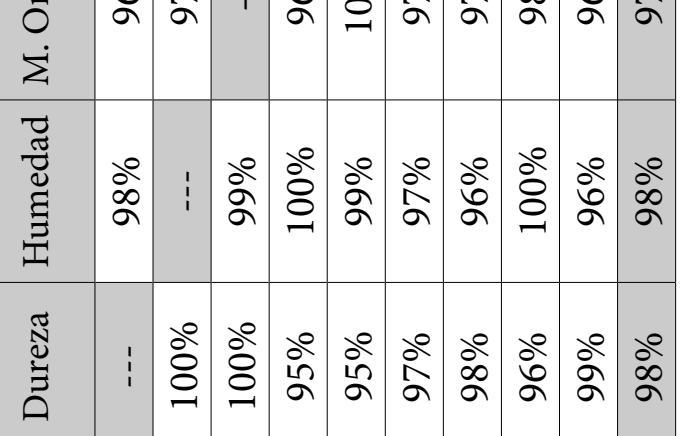

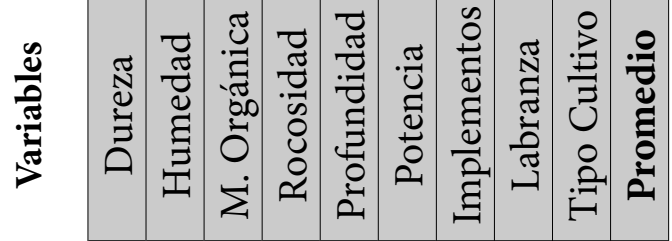

Tales relaciones entre variables se modelan en una gráfica de radio (Figura 9), la cual tiene como centro el $90 \%$ de efectividad y el $100 \%$ es el perímetro externo. Los datos no se dispersaron mucho, sino al contrario, se concentraron entre el $95 \%$ y el $100 \%$.

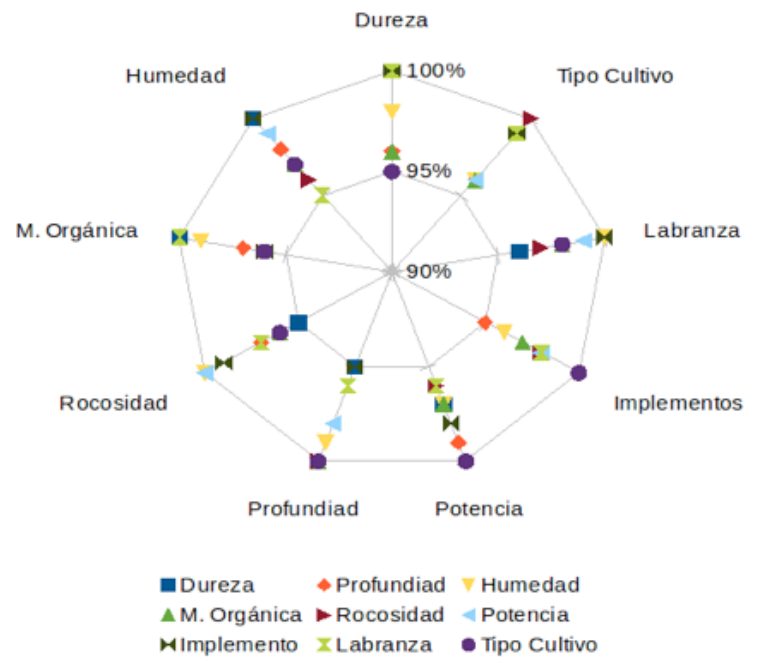

Figura 9. Precisión del modelo por cada variable cruzada, partiendo del $90 \%$ centro, hacia el $100 \%$.

\section{d. Aporte a las Ciencias Agronómicas}

Al momento de escribir el artículo, se encontró que la información acerca de técnicas de labranza agrícola mecanizada en El Salvador es muy limitada y dispersa. Los documentos encontrados en Internet sobre la temática, están orientados a otros países, con climas y suelos muy diferentes. Tampoco se encontraron guías técnicas condensadas o investigación científica aplicada a la labranza de suelos para el país. Por tales razones, el depósito de 
dicha información es cultural, depende de la experiencia y criterio de aquellos que trabajan en el campo operando maquinaria. Por tales motivos, el software SIMA constituyó un elemento innovador tanto para el área educativa de las Ciencias Agronómicas y para quienes se dedican a la agricultura. Éste no sólo recopila las prácticas tradicionales, sino que las modela con base en criterios técnicos, y ofrece recomendaciones concretas de apoyo para lo toma de decisiones de labranza de suelos antes de que se establezcan los cultivos. Los servicios del algoritmo pueden consumirse desde una interfaz web adaptativa, cuya URL es: http:// www.catolica.edu.sv/decanatos/iya/sima/

\section{e. Comparación con resultados de otros estudios}

Aunque no existe software alguno de este tipo, adecuado a las condiciones de El Salvador, existen otros proyectos en el extranjero que demuestran el éxito de diversos algoritmos matemáticos para el modelado de situaciones reales en el campo de la educación (Fuentes, 2006 y Schez, 2012), el modelado de disponibilidad de agua (Leao et. al, 2005; Muñoz y Grieser, 2006 y Martínez, 2015) y nutrientes en suelos cultivables (Mehrabian et al., 2006; Varbel et al., 2007 y Solie et al., 2012), el patrón de propagación de especies biológicas (Guindon y Gascuel, 2003 y Vargas, 2008); además de diversas aplicaciones que incorporan algoritmo para el procesamiento de información estratégica (Rodríguez, 2007; Calza- da, 2009; Gutiérrez, 2009; Mazzarra, 2011 y Jeong, 2014).

En todos esos estudios se demostró que un algoritmo de software puede ser una poderosa herramienta de apoyo para la toma de decisiones.

- Es posible reducir costos de cultivos mediante la creación de modelos de labranza para la toma oportuna de decisiones.

- Fue posible simular una amplia variedad de casos para su estudio.

- El uso mismo del software puede favorecer las competencias sobre resolución de problemas, aún en escenarios variantes (Martínez, 2015).

- Se posibilita el análisis predictivo de situaciones extremas o complejas.

\section{Conclusiones}

Para poder establecer apropiadamente un cultivo, se requiere que diversas variables cumplan con las condiciones apropiadas, tales como la humedad, la textura del suelo, el nivel de la capa rocosa, la cantidad de materia orgánica, entre otras. Para modelar planes de labranzas agrícolas para cultivos, se tomó en consideración no sólo las condiciones óptimas para cada variable, sino las relaciones entre ellas, tales como los implementos agrícolas a usar según la presencia de rocas y de humedad en el suelo. Luego, fue necesario 
determinar el número la cantidad de veces que debe repetirse cada labranza y la potencia requerida de un tractor. De acuerdo con umbrales económicos de la relación beneficio/costo, se determinó cuándo era recomendable alquilar o adquirir maquinaria propia. Se consideraron otras incidencias como los tiempos de espera entre labranza, de acuerdo con el contenido de humedad del suelo o la presencia de vientos que pudiesen causar erosión eólica, entre otras relaciones cruzadas entre las mismas variables.

La gran diversidad de datos que debió tomarse en cuenta para la elaboración del algoritmo y las complejas relaciones multivariadas conllevaron a la necesidad de establecer una base de datos, aunque si bien estática, pero con la capacidad de establecer consultas cruzadas entre la información, de forma eficiente.
El algoritmo en sí fue capaz de recibir los datos del modelo ingresado por el usuario desde la Web, validarlos mediante reglas de comparación, estimar las condiciones del suelo e iterar las cantidades de labranzas requeridas hasta encontrar una combinación que permitiese disponer de un sustrato suave, apto para ser cultivado.

Los resultados que el algoritmo fue capaz de proveer, pueden ser utilizados como referencia de técnicas de mecanización de labranza agrícola para El Salvador, tanto con fines educativos, o de apoyo para la toma de decisiones en emprendimientos agrícolas reales. Según la validación realizada, en la que se cuantificó cuán acertados eran los resultados, se obtuvo un satisfactorio nivel de $98 \%$.

\section{Referencias}

Alvarado, O., Jaramillo, R., Valverde, F. y Parra, R. (2011). Manejo de nutrientes por sitio específico en el cultivo de maíz bajo labranza de conservación para la provincia de Bolívar. Estacion Experimental Santa Catalina, Ecuador. Recuperado de https://books.google.com. sv/books?id=d3ozAQAAMAAJ\&lpg=PA13\&ots=JHwmD35AMa\&lr\&hl=es\&pg=PP1\#v=on epage $\& \mathrm{q}=$ tractor $\& \mathrm{f}=$ false

Borroto, O. (2005). Medidas de conservación para suelos potencialmente erosionables. Red cubana de ciencias. Recuperado de http://www. bibliociencias.cu/gsdl/collect/tesis/index/assoc/ HASH8ec4.dir/doc.pdf.

Calzada, L. (2009). El impacto de las herramientas de inteligencia de negocios en la toma de decisiones de los ejecutivos. International Journal of Good Conscience. Recuperado de http:// datateca.unad.edu.co/contenidos/206045/Unidad\%201\%20Inteligencia\%20de\%20Negocios/ Impactodelaherramientas\%20del Nenlosejecutivos.pdf 
Chaves, A. (2006). Mecanización agrícola ¿Deterioro o conservación del suelo? Revista Tecnología en Marcha. Recuperado de http://revistas.tec. ac.cr/index.php/tec_marcha/ article/view/24

Departamento de Ciencia de Suelos. (2010). Manejo de suelos en Wisconsin. Quinta Edición. Publicado por la Universidad de Wisconsin-Madison. Recuperado de http://www.soils.wisc. edu/extension/ pubs/A3588.pdf

Fuentes, L. (2006). Software educativo para la enseñanza de la Biología. Biblioteca Digital Revicyhluz. ISSN 1012-1587. Recuperado de http://200.74.222.178/index.php/opcion/article/ download/ 6334/6322

Glusac, D. (2008). El rol del material educativo digital en la enseñanza efectiva. Universidad de Novi Sad (Serbia). Recuperado de http://www.wseas.us/e-library/conferences/2008/spain/ diweb-miv/diweb-iv13.pdf

Guindon, S., y Gascuel, O. (2003). A simple, fast, and accurate algorithm to estimate large phylogenies by maximum likelihood. Recuperado de http://sysbio.oxfordjournals.org/content/52/5/696.full.pdf + html.

Gutierrez, O. (2009). Un enfoque multicentro para la toma de decisiones en la gestión de inventarios. Facultad de Ciencias Económicas y Empresariales de la Universidad de Oriente, Cuba. Recuperado de http://revistas.javeriana.edu.co/ index.php/cuadernos_admon/article/ view/3870/2839

Instituto Canadiense de Fosfato y Potasio, (2006). Manual de Fertilidad de Suelos. Publicado por Instituto Nacional de Nutrición Vegetal. ISBN 0-9629598-5-5.

Instituto Canadiense de Fosfato y Potasa, (2012). Preparing for the International Certified Crop Advisor. Décimo novena edición. Publicado por Instituto Nacional de Nutrición Vegetal.

Jeong, J.S., García-Moruno, L. y Hernández-Blanco, J. (2014) Un modelo web para la asistencia en la toma de decisiones en la integración de las construcciones rurales mediante planificación espacial multi-criterio. Informes de la Construcción. Recuperado de http://dx.doi.org/10.3989/ic.13.001

Kendal, K. (2012). Análisis y Diseño de Sistemas. Octava edición. México, Pearson Education. ISBN: 978-607-32-0577-1. 
Leao, T. P., da Silva, A. P., Perfect, E., y Tormena, C. A. (2005). An algorithm for calculating the least limiting water range of soils. Agronomy Journal. Recuperado de http//web.eps.utk.edu/ faculty/perfect/Papers/Leao\%20et\%20al\%20 (2005).pdf

Martínez, C. R. (2016). El software de entrenamiento para la detección visual de fitopatologías. Anuario de Investigación. Vol. 5 pp. 73-90; Ed. UNICAES editores

Mazzara, P. y Steinfeld, L. (2011). Despliegue y Depuración de Redes de Sensores Inalámbricos para Aplicaciones al Agro. Instituto de Ingeniería Eléctrica, Facultad de Ingeniería, Universidad de la República, Montevideo, Uruguay. Recuperado de https://iie.fing.edu.uy/publicaciones/2011/MSVSFOSBVG11/ MSVSFOSBVG11.pdf

Mehrabian, A. R. y Lucas, C. (2006). A novel numerical optimization algorithm inspired from weed colonization. Ecological informatics. Recuperado de https://www.researchgate.net/publication/222656762_Lucas_C_A_novel_numerical_optimization_algorithm_inspired_from_ weed_colonization_Ecological_Informatics_1_355-366

Mendoza, M. (2015). Labranza mecanizada en la productividad del cultivo de maíz h. Trueno. Escuela Superior Politécnica Agropecuaria de Manabí, “Manuel Félix López”, Ecuador. Recuperado de http://repositorio.espam.edu.ec/handle/ 42000/36

Muñoz, G. y Grieser, J. (2006). CLIMWAT 2.0 for CROPWAT. FAO de la ONU. Recuperado de: http://www.juergen-grieser.de/downloads/CLIMWAT_2.pdf

Reis, R. (2007). Desarrollo de Software Educativo. Publicado por International Journal of Education and Information Technologies. Recuperado de http://www.naun.org/multimedia/ NAUN/education information/eit-27.pdf

Rivera, P. (2015). El Laboreo De Los Suelos Agrícolas. Universidad Autónoma Agraria Antornio Narro. México. Recuperado de http://repositorio.uaaan. mx:8080/xmlui/handle/123456789/5684

Rodríguez, A. (2007) Integración de un S.I.G. con modelos de cálculo y optimización de rutas de vehículos CRVP y software de gestión de flotas. Universidad Politécnica De Valencia. Recuperado de http://revistadyo.com/index.php/dyo/article/view/50/50 
Schez, C. (2012). Diseño y desarrollo de software para laboratorios virtuales educativos con técnicas de procesamiento de lenguaje: Lecciones aprendidas en experimentos prácticos. Publicado por Journal of Research and Practice in Information Technology. Recuperado de http:// search.informit.com.au/documentSummary;dn=224660290474993;res=IELHSS

Solie, J. B., Monroe, A. D., Raun, W. R. y Stone, M. L. (2012). Generalized algorithm for variable-rate nitrogen application in cereal grains. Agronomy journal. Recuperado de http:// www.nue.okstate.edu/Index_Publications/GA_pdt.pdf

Vargas, B.; Corral, J. y otros (2008). Uso y aplicación de los simuladores de crecimiento forestal en la toma de decisiones silviculturales. Revista Forestal Latinoamericana. Recuperado de http://www.saber.ula.ve/bitstream/123456789/33374/1/articulo2.pdf

Varvel, G. E., Wilhelm, W. W., Shanahan, J. F. y Schepers, J. S. (2007). An algorithm for corn nitrogen recommendations using a chlorophyll meter based sufficiency index. Agronomy Journal. Recuperado de http://digitalcommons. unl.edu/cgi/viewcontent.cgi? article=1179 \&context=usdaarsfacpub 